\title{
Kolmogorov's Extension Theorem for Infinite Measures
}

\author{
By
}

Yasuo YAMASAKI

\begin{abstract}
We consider the extension problem of a self-consistent family of infinite measures to a completely additive measure. For probability measures, Kolmogorov's extension theorem assures that the extension is uniquely possible. Our results are as follows:

(a) For $\sigma$-finite measures, we can reduce the problem to the case of probability measures, so that the extension is uniquely possible. As an application, on an infinite dimensional vector space we can construct such a measure that is invariant both under rotations and homotheties with respect to the origin. It is obtained as the limit of $n$ dimensional measure:

$$
\frac{1}{|x|^{n}} d x_{1} d x_{2} \cdots d x_{n}
$$

Also we shall discuss about the Lorentz invariant measure on an infinite dimensional space.

(b) If measures are not $\sigma$-finite, under the additional condition (EG) in $\$ 6$, the extension is possible but not unique. We shall mention about the largest and the smallest extension. As an application, we can consider the symbolic representation of a flow $\left\{T_{t}\right\}$ defined on an infinite measure space $X$, namely constructing an appropriate product space $W^{\boldsymbol{R}}$ and an appropriate measure on $W^{\boldsymbol{R}}, T_{t}$ on $X$ is represented by a shift $S_{t}$ on H $\boldsymbol{R}: w(\cdot) \rightarrow w(\cdot+t)$.

$\S 1$ Kolmogorov's extension theorem

$\S 2$ Reduction to finite measures

\$3 Rotationally invariant measure

$\S 4(0, \infty)$-type measures

\$5 Lorentz invariant measure

$\$ 6$ Non $\sigma$-finite case

$\$ 7 \quad \sigma$-finite plus essentially infinite case

$\$ 8$ Symbolic representation of flows
\end{abstract}

\section{$\S 1$. Kolmogorov's Extension Theorem}

Let $\Omega$ be a set, and $\mathfrak{B}$ be a $\sigma$-ring of subsets of $\Omega$. The pair $\{\Omega, \mathfrak{B}\}$

Received December 27, 1973. 
is called a measurable space.

For a sequence of measurable spaces $\left\{\Omega^{(k)}, \mathfrak{B}^{(k)}\right\}$, we define the product measurable space $\{\Omega, \mathfrak{B}\}$ as follows:

$$
\begin{aligned}
& \Omega= \prod_{k=1}^{\infty} \Omega^{(k)} \\
& \mathfrak{B}= \text { the } \sigma \text {-ring generated by } \bigcup_{k=1}^{\infty} p^{(k)^{-1}\left(\mathfrak{B}^{(k)}\right)} \text {, where } p^{(k)} \text { is the } \\
& \quad \text { projection } \Omega \rightarrow \Omega^{(k)} .
\end{aligned}
$$

Similarly, we shall define the finite product $\left\{\Omega_{n}, \mathfrak{B}_{n}\right\}$.

$$
\begin{aligned}
\Omega_{n} & =\prod_{k=1}^{n} \Omega^{(k)} \\
\mathfrak{B}_{n} & =\text { the } \sigma \text {-ring generated by } \bigcup_{k=1}^{n} p^{(k)^{-1}}\left(\mathfrak{B}^{(k)}\right) .
\end{aligned}
$$

Let $\mu_{n}$ be a $\sigma$-additive measure defined on $\mathfrak{B}_{n}$. A sequence of measures $\left\{\mu_{n}\right\}$ is called self-consistent if $\mu_{n}\left(E_{n}\right)=\mu_{m}\left(p_{m n}^{-1}\left(E_{n}\right)\right)$ for any $m>n$ and any $E_{n} \in \mathfrak{B}_{n}$, where $p_{m n}$ is the projection $\Omega_{m}=\Omega_{n} \times \prod_{k=n+1}^{m} \Omega^{(k)} \rightarrow \Omega_{n}$. Such a sequence $\left\{\mu_{n}\right\}$ defines a finitely additive measure $\mu$ on $\Omega$ as follows:

Let $p_{n}$ be the projection $\Omega=\Omega_{n} \times \prod_{k=n+1}^{\infty} \Omega^{(k)} \rightarrow \Omega_{n}$, then $\mathfrak{B}$ is also the $\sigma$-ring generated by $\mathfrak{F}=\bigcup_{n=1}^{\infty} p_{n}^{-1}\left(\mathfrak{B}_{n}\right)$. If $E \in \mathfrak{F}$, we have $E=p_{n}^{-1}\left(E_{n}\right)$ for some $n$ and for some $E_{n} \in \mathfrak{B}_{n}$, and we may put $\mu(E)=\mu_{n}\left(E_{n}\right)$. This defines $\mu$ uniquely on $\mathfrak{F}$ because of self-consistency condition of $\left\{\mu_{n}\right\}$. The obtained $\mu$ is a finitely additive measure on $\mathfrak{F}$, while $\sigma$-additive on $p_{n}^{-1}\left(\mathfrak{B}_{n}\right)$ for each $n$.

In this paper, we shall discuss the extendability of $\mu$ to a $\sigma$-additive measure on $\mathfrak{B}$.

If each $\Omega^{(k)}$ is the real line $\mathbb{R}^{1}$ and $\mathfrak{B}^{(k)}$ is the $\sigma$-ring of all Borel sets, (so that $\Omega_{n}=\mathbb{R}^{n}$ and $\mathfrak{B}_{n}$ is the family of all Borel sets of $\mathbb{R}^{n}$ ), and if each $\mu_{n}$ is a probability measure, Kolmogorov proved such an extension is uniquely possible [1].

Bochner generalized the situation from the infinite product of measurable spaces to the projective limit of measurable spaces [2]. In Bochner's formulation, let $\left\{\Omega_{n}, \mathfrak{B}_{n}\right\}$ be a sequence of measurable spaces, and for $m>n$ let $p_{m n}$ be a measurable onto mapping from $\Omega_{m}$ to $\Omega_{n}$. We assume 
the condition $p_{m n}=p_{m k} \circ p_{k n}$ for any $m>k>n$.

Let $\mu_{n}$ be a $\sigma$-additive measure on $\mathfrak{B}_{n}$. A sequence of measures $\left\{\mu_{n}\right\}$ is called self-consistent if

$$
\mu_{n}\left(E_{n}\right)=\mu_{m}\left(p_{m n}^{-1}\left(E_{n}\right)\right) \text { for any } m>n \text { and any } E_{n} \in \mathfrak{B}_{n} .
$$

Let $\Omega$ be a subset of $\prod_{n=1}^{\infty} \Omega_{n}$ such that

$$
\left(\omega_{1}, \omega_{2}, \ldots, \omega_{n}, \ldots\right) \in \Omega \Leftrightarrow \omega_{n}=p_{m n}\left(\omega_{m}\right) \text { for any } m>n
$$

Let $p_{k}$ be the restriction on $\Omega$ of the projection $\pi_{k}: \prod_{k=1}^{\infty} \Omega_{k} \rightarrow \Omega_{k}$. Then, put $\mathfrak{F}=\bigcup_{k=1}^{\infty} p_{k}^{-1}\left(\mathfrak{B}_{k}\right)$, and $\mathfrak{B}=$ the $\sigma$-ring generated by $\mathfrak{F}$. Evidently, a self-consistent sequence of measures $\left\{\mu_{n}\right\}$ defines a finitely additive measure $\mu$ on $\mathfrak{F}$.

It is easily seen that Bochner's formulation is applicable to the infinite product of measurable spaces as a special case.

In Bochner's formulation, the extendability of $\mu$ to a $\sigma$-additive measure on $\mathfrak{B}$ is in question. If each $\Omega_{n}$ is a locally compact, $\sigma$-compact metric space and $\mathfrak{B}_{n}$ is the $\sigma$-ring of all Borel sets of $\Omega_{n}$, we can prove the extendability and its uniqueness for a self-consistent family of probability measures. (Of course, this result can be applied for a special case of infinite product measurable space).

The obtained $\{\Omega, \mathfrak{B}\}$ is called the projective limit measurable space of $\left\{\Omega_{n}, \mathfrak{B}_{n}\right\}$, and $\{\Omega, \mathfrak{B}, \mu\}$ is called the projective limit measure space of $\left\{\Omega_{n}, \mathfrak{S}_{n}, \mu_{n}\right\}$.

Instead of locally compactness and $\sigma$-compactness, if we assume that each $\Omega_{n}$ is a complete metric space, the extendability and its uniqueness is proved for a family of probability measures. (for instance, c.f. [3]).

Above results are still valid even if we replace a sequence of measures by a family of measures indexed by elements of an ordered set.

\section{§2. Reduction to Finite Measures}

From this section on, we assume that the family of measurable spaces $\left\{\Omega_{n}, \mathfrak{B}_{n}\right\}$ is such that any self-consistent family of probability measures 
$\left\{\mu_{n}\right\}$ can be extended uniquely to a $\sigma$-additive measure. For instance, it is sufficient to suppose that $\Omega_{n}$ is either a locally compact and $\sigma$-compact or a complete metric space and that $\mathfrak{B}_{n}$ is the $\sigma$-ring of all Borel sets of $\Omega_{n}$.

1) Proposition 2.1. For a self-consistent family of finite measures $\left\{\mu_{n}\right\}$, the extension is uniquely possible.

Proof Put $c_{n}=\mu_{n}\left(\Omega_{n}\right)<\infty$. From the self-consistency condition, $c_{n}$ does not depend on $n$, so we put this common value as $c$. Then, the measures $\bar{\mu}_{n}=\frac{1}{c} \mu_{n}$ form a self-consistent family of probability measures, so that $\left\{\bar{\mu}_{n}\right\}$ can be extended uniquely to a $\sigma$-additive measure $\bar{\mu}$. It is evident that the $\sigma$-additive measure $c \bar{\mu}$ is a unique extension of measures $\left\{\mu_{n}\right\}$.

2) Let $\left\{\mu_{n}\right\}$ be a self-consistent family of infinite measures. As defined in $\S 1$, we put $\mathfrak{F}=\bigcup_{n} p_{n}^{-1}\left(\mathfrak{B}_{n}\right)$ and $\mathfrak{B}=$ the $\sigma$-ring generated by $\mathfrak{F}$. Then, the family $\left\{\mu_{n}\right\}$ defines a finitely additive measure $\mu$ on $\mathfrak{F}$, as explained in $\S 1$.

Suppose that $A \in \mathfrak{F}$ and $\mu(A)<\infty$, namely suppose that $A=p_{n}^{-1}\left(A_{n}\right)$, $A_{n} \in \mathfrak{B}_{n}$ and $\mu_{n}\left(A_{n}\right)<\infty$, then the measures

$$
\begin{aligned}
\mu_{k}^{(A)}\left(E_{k}\right)= & \mu_{m}\left(p_{m k}^{-1}\left(E_{k}\right) \cap p_{m n}^{-1}\left(A_{n}\right)\right) \\
& \text { for } \quad E_{k} \in \mathfrak{B}_{k}, \quad m \geqq \operatorname{Max}(n, k)
\end{aligned}
$$

form a self-consistent family of finite measures, so that $\left\{\mu_{k}^{(A)}\right\}$ can be extended uniquely to a $\sigma$-additive measure $\mu^{(A)}$ on $\mathfrak{B}$.

For $A^{\prime} \supset A$, it is easily seen that we have

$$
\begin{aligned}
& \mu^{(A)}(E)=\mu^{\left(A^{\prime}\right)}(E \cap A) \text { for } \quad E \in \mathfrak{B} \\
& =\mu(E \cap A) \quad \text { for } \quad E \in \mathfrak{F} .
\end{aligned}
$$

3) Put $\mathfrak{B}_{0}=\left\{B \in \mathfrak{B} ;{ }^{\exists} A_{1}, A_{2}, \ldots \in \mathfrak{F}, \mu\left(A_{n}\right)<\infty, B \subset \bigcup_{n=1}^{\infty} A_{n}\right\}$. In this definition, $\left\{A_{n}\right\}$ can be supposed to be mutually disjoint. We shall always impose this additional condition on $\left\{A_{n}\right\}$.

For $B \in \mathfrak{B}_{0}$, we define a $\sigma$-additive measure $\mu^{(B)}$ as follows:

$$
\mu^{(B)}(E)=\sum_{n=1}^{\infty} \mu^{\left(A_{n}\right)}(E \cap B) \quad \text { for } \quad E \in \mathfrak{B} .
$$


This $\mu^{(B)}$ is a $\sigma$-additive measure because every term in the right side is so.

Now, we shall remark that the measure $\mu^{(B)}$ does not depend on the choice of $\left\{A_{n}\right\}$. Suppose that $B \subset \cup_{n} A_{n}$ and $B \subset \cup_{n} A_{n}^{\prime}$. Then, from the $\sigma$-additivity of the measure $\mu^{\left(A_{n}\right)}$, we have

$$
\mu^{\left(A_{n}\right)}(E \cap B)=\sum_{k=1}^{\infty} \mu^{\left(A_{n}\right)}\left(E \cap B \cap A_{k}^{\prime}\right),
$$

but in virtue of (2.2), the right side is equal to

$$
\sum_{k=1}^{\infty} \mu^{\left(A_{n}\right)}\left(E \cap B \cap A_{k}^{\prime} \cap A_{n}\right)=\sum_{k=1}^{\infty} \mu^{\left(A_{k}^{\prime} \cap A_{n}\right)}(E \cap B)
$$

Thus, the right side of (2.3) is equal to

$$
\sum_{n=1}^{\infty} \sum_{k=1}^{\infty} \mu^{\left(A_{k}^{\prime} \cap A_{n}\right)}(E \cap B)
$$

This assures the independence of $\mu^{(B)}$ from the choice of $\left\{A_{n}\right\}$.

If $B, B^{\prime} \in \mathfrak{B}_{0}$ and $B \subset B^{\prime}$, we have

$$
\mu^{(B)}(E)=\mu^{\left(B^{\prime}\right)}(E \cap B) \text { for } E \in \mathfrak{B} .
$$

But even if $E \cap B \in \mathfrak{F}$, there is a question whether the right side of (2.4) is equal to $\mu(E \cap B)$ or not. We shall discuss this point in 5).

4) If the measures $\left\{\mu_{n}\right\}$ can be extended to a $\sigma$-additive measure $\bar{\mu}$ on $\mathfrak{B}$, we must have from (2.2)

$$
\begin{aligned}
\bar{\mu}(E \cap A) & =\mu^{(A)}(E) \\
& \text { for } \quad E \in \mathfrak{B}, \quad A \in \mathfrak{F}, \quad \mu(A)<\infty .
\end{aligned}
$$

So, also for $B \in \mathfrak{B}_{0}$ we have

$$
\begin{aligned}
\bar{\mu}(E \cap B)=\sum_{n=1}^{\infty} \bar{\mu}\left(E \cap B \cap A_{n}\right) & =\sum_{n=1}^{\infty} \mu^{\left(A_{n}\right)}(E \cap B) \\
& =\mu^{(B)}(E) .
\end{aligned}
$$

Proposition 2.2. If $\Omega \in \mathfrak{B}_{0}$, the extension (if possible) is unique.

Proof From (2.5) we have

$$
\bar{\mu}(E)=\mu^{(\Omega)}(E) \quad \text { for any } \quad E \in \mathfrak{B},
$$


thus we have $\bar{\mu}=\mu^{(\Omega)}$. (q.e.d.)

The possibility of extension depends on whether $\mu^{(\Omega)}$ is identical with $\mu$ or not on $\mathfrak{F}$. Namely, the condition:

$$
\mu^{(\Omega)}(E)=\mu(E) \text { for } \quad E \in \mathfrak{\mho}
$$

is necessary and sufficient for the unique extension of $\left\{\mu_{n}\right\}$ to a $\sigma$-additive measure. (2.7) means

$$
\mu(E)=\sum_{n=1}^{\infty} \mu\left(E \cap A_{n}\right) \quad \text { for } \quad E \in \mathfrak{F}
$$

where $\left\{A_{n}\right\}$ is such that $A_{n} \in \mathfrak{F}, \mu\left(A_{n}\right)<\infty$ and $\Omega=\bigcup_{n=1}^{\infty} A_{n}$.

If all $A_{n}$ can be chosen in $p_{m}^{-1}\left(\mathfrak{B}_{m}\right)$ for fixed $m$, the condition $(2.7)^{\prime}$ is satisfied in virtue of the $\sigma$-additivity of $\mu_{m}$. Therefore we have

Theorem 1. If one of measures $\left\{\mu_{n}\right\}$, say $\mu_{n_{0}}$, is $\sigma$-finite, then $\left\{\mu_{n}\right\}$ can be extended uniquely to a $\sigma$-additive measure on $\mathfrak{B}$.

For the case where $\Omega_{n}$ is locally compact and $\sigma$-compact, Y. Okabe proved the above theorem [4], modifying the proof of Kolmogorov's extension theorem. Here, we have reduced the theorem to the result of Kolmogorov.

5) In the last part of 3), we remarked that

$$
\begin{aligned}
& \mu^{(B)}(E)=\mu(E \cap B) \\
& \text { for } \quad E \in \mathfrak{B}, \quad B \in \mathfrak{B}_{0}, \quad E \cap B \in \mathfrak{\mho}
\end{aligned}
$$

can not be proved. This equality is a necessary condition for the extendbility of $\left\{\mu_{n}\right\}$ as seen in (2.5).

(2.8) is equivalent to

$$
\mu(E)=\sum_{n=1}^{\infty} \mu\left(E \cap A_{n}\right) \quad \text { for } \quad E \in \mathfrak{F} \cap \mathfrak{B}_{0},
$$

where $\left\{A_{n}\right\}$ is such that $A_{n} \in \mathfrak{F}, \mu\left(A_{n}\right)<\infty$ and $E \subset \bigcup_{n=1}^{\infty} A_{n}$. Therefore, it is a generalization of $(2.7)^{\prime}$ to the case $\Omega \notin \mathfrak{B}_{0}$.

Since $\mu$ is not $\sigma$-additive on $\mathfrak{F}$, (though $\sigma$-additive on each $p_{m}^{-1}\left(\mathfrak{B}_{m}\right)$ ), $(2.7)^{\prime}$ or $(2.8)^{\prime}$ is not a direct consequence. Up to the present time, we can not prove it, nor can not give a counter-example. 
Let $\Omega_{n}=N$ (the set of all positive integers), $\mathfrak{B}_{n}=\{\{1\},\{2\}, \ldots,\{n\}$, $\{n+1, n+2, \ldots\}\}, p_{m n}$ be the identity mapping, and $\mu_{n}(\{k\})=0$ for $k \leqq n$, $\mu_{n}(\{n+1, n+2, \ldots\})=\infty$. Then, we have $\mu(\boldsymbol{N})=\infty$ but $\mu(\{k\})=0$ for any $k$, so it gives a counter-example. But for this $\left\{\Omega_{n}, \mathfrak{B}_{n}\right\}$, even a family of probability measures can not always be extended.

It is urgent to give a counter-example (if exists) of $(2.7)^{\prime}$ for the family $\left\{\Omega_{n}, \mathfrak{B}_{n}\right\}$ which allows a unique extension for probability measures.

Remark If $\mu(E)<\infty,(2.7)^{\prime}$ or (2.8) is trivially satisfied, because in both hand side $\mu$ can be replaced by $\mu^{(E)}$ which is $\sigma$-additive. $(2.8)^{\prime}$ is equivalent to the following condition:

If $E \in \mathfrak{F} \cap \mathfrak{B}_{0}, \mu(E)=\infty$, then for any $N>0$ there exists such $F \in \mathfrak{F}$ that satisfies $F \subset E$ and $N<\mu(F)<\infty$.

\section{§3. Rotationally Invariant Measure}

In the next three sections we shall give some examples and remarks on the results in $\S 2$, and we shall continue the general discussions on extendability in $\S 6$.

1) Let $\mu_{n}$ be a measure on $\boldsymbol{R}^{n}$ defined as follows:

$$
d \mu_{n}=\frac{1}{|x|^{n}} d x_{1} \ldots d x_{n}
$$

where $|x|=\sqrt{x_{1}^{2}+\ldots+x_{n}^{2}}$. On the space $\boldsymbol{R}^{n}$, it is the unique (except a constant factor) measure which is invariant both under rotations and homotheties with respect to the origin.

Since we have

$$
\begin{aligned}
\int_{-\infty}^{\infty} \frac{d x_{n+1}}{\left(|x|^{2}+x_{n+1}^{2}\right)^{\frac{n+1}{2}}} & =\frac{2}{|x|^{n}} \int_{0}^{\frac{\pi}{2}} \cos ^{n-1} \theta d \theta \\
& =\frac{\sqrt{\pi}}{|x|^{n}} \frac{\Gamma\left(\frac{n}{2}\right)}{\Gamma\left(\frac{n+1}{2}\right)},
\end{aligned}
$$

the family of measure $\left\{c_{n} \mu_{n}\right\}$ where 


$$
c_{n}=\frac{\Gamma\left(\frac{n}{2}\right)}{\pi^{n / 2}}
$$

forms a self-consistent family of $\sigma$-finite measures.

Therefore, on the infinite product space $\boldsymbol{R}^{\infty}$, we can define uniquely a $\sigma$-finite, $\sigma$-additive measure $\mu$ whose projection on $\boldsymbol{R}^{n}$ is $c_{n} \mu_{n}$.

2) $\boldsymbol{R}^{\infty}$ is the space of all real sequences, and containes $\boldsymbol{R}_{0}^{\infty}$ as a subspace where

$$
\mathbb{R}_{0}^{\infty}=\left\{\left(x_{n}\right) \in \boldsymbol{R}^{\infty} ; \quad{ }^{\exists} N, n \geqq N \Rightarrow x_{n}=0\right\} .
$$

Let $\left(l^{2}\right)$ be the space of square-summable real sequences. Evidently we get $\boldsymbol{R}_{0}^{\infty} \subset\left(l^{2}\right) \subset \boldsymbol{R}^{\infty}$. Let $\mathrm{O}_{\infty}$ be the group of orthogonal operators of $\left(l^{2}\right)$ which keep $\boldsymbol{R}_{0}^{\infty}$ invariant. Identifying $U$ with $U^{-1 *}$, $\mathrm{O}_{\infty}$ can be regarded as a transformation group on $\boldsymbol{R}^{\infty}$, the dual space of $\boldsymbol{R}_{0}^{\infty}$.

Since the measure $\mu_{n}$ defined in (3.1) is rotationally invariant, the family $\left\{c_{n} \mu_{n}\right\}$ defines an $\mathrm{O}_{\infty}$-invariant measure on $\mathfrak{\mho}=\bigcup_{n} p_{n}^{-1}\left(\mathfrak{B}_{n}\right)$, and the extension being unique, the extended $\sigma$-additive measure must be $\mathrm{O}_{\infty}$-invariant on $\mathfrak{B}$, the smallest $\sigma$-ring which containes $\mathfrak{F}$.

In the same way, $\mu$ is invariant under homotheties with respect to the origin, namely

$$
\mu(E)=\mu(\lambda E) \quad \text { for } \quad E \in \mathfrak{B}, \lambda>0 .
$$

3) Let $H$ be a real Hilbert space, and $L$ be a locally convex space which is imbedded densely and nuclearly in $H$.

Let $\mathrm{O}_{L}$ be the group of orthogonal operators of $H$ which keep $L$ invariant and act homeomorphically on $L$. Then, $\mathrm{O}_{L}$ can be regarded also as a transformation group on $L^{*}$, the dual space of $L$. (c.f. [5])

Choosing a CONS (=complete orthonormal system) $\left\{e_{n}\right\}$ of $H$ in $L, \mathbb{R}_{0}^{\infty}$ can be imbedded in $L$ and we have

$$
\boldsymbol{R}_{\mathbf{0}}^{\infty} \subset L \subset H \simeq H^{*} \subset L^{*} \subset \boldsymbol{R}^{\infty} .
$$

Then, the measure $\mu$ obtained in 1) lies on $L^{*}$, namely we have

$$
\mu\left(\boldsymbol{R}^{\infty}-L^{*}\right)=0,
$$


and $\mu$ is $O_{L}$-invariant on $L^{*}$.

Using the infinite dimensional gaussian measure $g_{c}$ (with variance $c^{2}$ ) on $L^{*}$, we have

$$
\mu(E)=\int_{0}^{\infty} \frac{2}{c} g_{c}(E) d c
$$

From (3.6) also, we see the invariance of $\mu$ both under rotations and homotheties. The proof of (3.6) is as follows:

Projection onto $\mathbb{R}^{n}$ gives

$$
\begin{aligned}
& \mu \longrightarrow c_{n} \mu_{n}=\frac{c_{n}}{|x|^{n}} d x_{1} \ldots d x_{n} \\
& g_{c} \longrightarrow \frac{1}{(2 \pi)^{n / 2} c^{n}} \exp \left[-\frac{|x|^{2}}{2 c^{2}}\right] d x_{1} \ldots d x_{n}
\end{aligned}
$$

so the equality

$$
\begin{aligned}
\frac{1}{(2 \pi)^{n / 2}} \int_{0}^{\infty} \frac{2}{c^{n+1}} \exp \left[-\frac{|x|^{2}}{2 c^{2}}\right] d c \\
=\frac{1}{(2 \pi)^{n / 2}} \frac{1}{|x|^{n}} \int_{0}^{\infty} 2 s^{n-1} \exp \left(-\frac{s^{2}}{2}\right) d s \\
=\frac{\Gamma\left(\frac{n}{2}\right)}{\pi^{n / 2}} \frac{1}{|x|^{n}}=\frac{c_{n}}{|x|^{n}}
\end{aligned}
$$

assures the validity of (3.6).

4) On the space $L^{*}$, any $\mathrm{O}_{L}$-invariant probability measure is necessarily a superposition of gaussian measures $g_{c}$, as proved in [5].

Let $\mu$ be an $\mathrm{O}_{L}$-invariant $\sigma$-finite measure on $L^{*}$. Then, there exist such $E_{n}$ that $E_{n} \in \mathfrak{B}, \mu\left(E_{n}\right)<\infty$ and $\bigcup_{n=1}^{\infty} E_{n}=L^{*}$. If we assume that each $E_{n}$ can be chosen to be $O_{L}$-invariant, the measure $\mu$ is also a superposition of gaussian measures, namely

$$
\mu(E)=\int_{0}^{\infty} g_{c}(E) d m(c) \text { for } \quad \forall E \in \mathfrak{B},
$$

because the restriction of $\mu$ on $E_{n}$ is an $O_{L}$-invariant finite measure so that it is a superposition of gaussian measures.

Moreover, if $\mu$ is invariant under homotheties, the measure $m(c)$ in 
(3.7) must be

$$
d m(c)=\frac{a}{c} d c \text { for some constant } \quad a>0
$$

Therefore, the measure $\mu$ obtained in 1 ) is the unique (except a constant factor) measure which is invariant both under rotations and homotheties.

\section{$\S 4 . \quad(0, \infty)$-type Measures}

1) In $\S 6$, we shall prove that any self-consistent family of measures $\left\{\mu_{n}\right\}$ satisfying $(2.8)^{\prime}$ can be extended to a $\sigma$-additive measure on $\mathfrak{B}$ and that the extension is unique on $\mathfrak{B}_{0}$ defined in $\S 2,3$ ).

If $\Omega \notin \mathfrak{B}_{0}$, the extension is not necessarily unique on $\mathfrak{B}$, but there exists the largest extension $\tilde{\mu}$ which is not $\sigma$-finite. However, this does not mean that $\left\{\mu_{n}\right\}$ can not be extended to a $\sigma$-additive, $\sigma$-finite measure, because some extension may be $\sigma$-finite even if $\Omega \notin \mathfrak{B}_{0}$.

We shall explain this in other words. Let $\mu$ be the finitely additive measure defined by $\left\{\mu_{n}\right\}$. Then, $\Omega \notin \mathfrak{B}_{0}$ means that $\Omega$ can not be covered by a countable union of sets in $\mathfrak{F}$ with finite $\mu$-measures. Even if we assume so, extending $\mu$ to a $\sigma$-additive measure $\bar{\mu}$ on $\mathfrak{B}, \Omega$ may be covered by a countable union of sets in $\mathfrak{B}$ with finite $\bar{\mu}$-measures.

In 3 ), 4) and 6) of this section, we shall give examples illustrating this situation.

2) A measure is called to be $(0, \infty)$-type if the measure of any measurable set is 0 or $\infty$. Especially, it is called to be essentially infinite if the measure of non-empty measurable set is always $\infty$.

For a self-consistent family of $(0, \infty)$-type measures $\left\{\mu_{n}\right\}$, the largest extension $\tilde{\mu}$ is also $(0, \infty)$-type. But the $\sigma$-ring $\mathfrak{B}_{0}$, on which the extension is unique, consists of null-sets only. In other words, the family $\left\{\mu_{n}\right\}$ can determine its extension almost nowhere. In this sense, we must conclude that to a family of $(0, \infty)$-type measures we can not attribute any limit measure. (c.f. 5) of this section).

We shall remark that even a family of $(0, \infty)$-type measures can be extended to a $\sigma$-finite measure. See examples in 3), 4) and 6). 
3) Let $\mathbb{Z}$ be the set of all integers, and $\mathbb{Z}^{\infty}$ be the infinite product of $\mathbb{Z}$. Consider a subset $\mathbb{Z}_{0}^{\infty}$ :

$$
\mathbb{Z}_{0}^{\infty}=\left\{\left(n_{k}\right) ;{ }^{\exists} K>0, k \geqq K \Rightarrow n_{k}=0\right\} .
$$

Remark that $\mathbb{Z}_{\mathbf{0}}^{\infty}$ is a countable set.

Regard $\mathbb{Z}$ as a measurable space, supposing that every subset is measurable. Then, we can regard $\mathbb{Z}^{\infty}$ also as a measurable space, imposing the structure of the infinite product measurable space of $\mathbb{Z}$. (c.f. $\S 1$ ).

Now, for a measurable subset $E$ of $\mathbb{Z}^{\infty}$. we shall define $\bar{\mu}(E)$ as follows:

$$
\bar{\mu}(E)=\text { number of points of } E \cap \mathbb{Z}_{0}^{\infty} .
$$

Evidently, $\bar{\mu}$ is a $\sigma$-additive, $\sigma$-finite measure on the measurable space $\mathbb{Z}^{\infty}$.

For any fixed element $\left(n_{1}, n_{2}, \ldots, n_{m}\right)$ of $\mathbb{Z}^{m}$, it is easily seen that

$$
p_{m}^{-1}\left(n_{1}, n_{2}, \ldots, n_{m}\right) \cap \mathbb{Z}_{0}^{\infty} \text { is an infinite set, }
$$

so the projection of $\bar{\mu}$ on $\mathbb{Z}^{m}$ is the essentially infinite measure. In other words, $\bar{\mu}$ is a $\sigma$-finite extension of a family of essentially infinite measures.

4) Let $\mathbb{R}^{+}$be the set of positive real numbers. For $x \in \mathbb{R}^{+}$, consider the binary expansion $x=\sum_{k=-\infty}^{\infty} \varepsilon_{k} 2^{k}$ where $\varepsilon_{k}=0$ or 1 . (Eventually $\varepsilon_{k}=0$ for sufficiently large $k$ ). Let $\varphi$ be the mapping which maps $x \in \mathbb{R}^{+}$ to $\left(\varepsilon_{k}(x)\right) \in\{0,1\}^{z}$. By this mapping, the Lebesgue measure on $\boldsymbol{R}_{+}$ is mapped to a measure $\bar{\mu}$ on $\{0,1\}^{z}$. (Though $\varphi(x)$ is not determined uniquely on a countable subset of $\mathbb{R}^{+}, \varphi$ determines $\bar{\mu}$ uniquely because the Lebesgue measure of a countable set is zero).

For any fixed element $\left(a_{1}, \alpha_{2}, \ldots, a_{m}\right)$ of $\{0,1\}^{m}$ and for any set of $m$ integers $\left\{k_{1}, k_{2}, \ldots, k_{m}\right\}$, the Lebesgue measure of the set:

$$
A_{\alpha_{1}, \alpha_{2}, \ldots, \alpha_{m} ; k_{1}, k_{2}, \ldots, k_{m}}=\left\{x \in \mathbb{R}^{+} ; \varepsilon_{k_{j}}(x)=a_{j} .1 \leqq j \leqq m\right\}
$$

is infinite, because this set is a countable union of intervals of same lengths.

Therefore, the projection of $\bar{\mu}$ on a finite product space of $\{0,1\}$ becomes the essentially infinite measure. On the other hand, since the Lebesgue measure is $\sigma$-finite, the measure $\bar{\mu}$ is also $\sigma$-finite. So, this gives another example of a $\sigma$-finite extension of a family of essentially infinite measures. 
5) If we try to construct on $\mathbb{R}^{\infty}$ an analogue of the Lebesgue measure, it seems natural to consider the family of $n$-dimensional Lebesgue measure. But unfortunately this family is not self-consistent.

For any finite interval $(a, b)$ in $\mathbb{R}^{1}$, the two-dimensional set $(a, b) \times \mathbb{R}^{1}$ has infinite area. Generally, the inverse of projection maps a set of finite measure in the lower dimensional space to a set of infinite measure in the higher dimensional space. (In other words, the ratio of normalizing constants $\left\{c_{n}\right\}$ is infinity. c.f. (3.3)).

Therefore, Lebesgue measure-like family is the family of $(0, \infty)$-type measures. Namely, we must consider the following measure $\mu_{n}$ on $\mathbb{R}^{n}$ :

$$
\begin{array}{ll}
\mu_{n}(E)=\infty & \text { if the Lebesgue measure of } E>0 \\
\mu_{n}(E)=0 & \text { if the Lebesgue measure of } E=0 .
\end{array}
$$

This is a result of the request that $\mu_{n}$ must be the projection of higher dimensional Lebesgue measure.

However, as remarked in 2$)$, the family of $(0, \infty)$-type measures determines its limit measure almost nowhere. So we must admit the failure of our attempt to obtain an $\infty$-dimensional analogue of the Lebesgue measure by our method.

Remark that on the space $\mathbb{R}^{\infty}$, there exists such a measure whose projection on any one-dimensional space is one-dimensional Lebesgue measure. For instance, it is sufficient to consider the Lebesgue measure lying on the diagonal:

$$
\Delta=\left\{(x, x, \ldots) ; x \in \mathbb{R}^{1}\right\} \subset \mathbb{R}^{\infty} .
$$

More generally, for a family of Lebesgue measure preserving one-to-one mappings $\left\{T_{n}\right\}$, the set:

$$
\Delta_{\{T\}}=\left\{\left(x_{1}, x_{2}, \ldots\right) ; x_{n}=T_{n} x, x \in \mathbb{R}^{1}\right\} \subset \mathbb{R}^{\infty}
$$

allows the one-dimensional Lebesgue measure on it.

However, two-dimensional projection of such a measure is not twodimensional Lebesgue measure.

6) Finally, we shall construct a $\sigma$-finite measure $\bar{\mu}$ which is an extension of $\left\{\mu_{n}\right\}$ defined in (4.3). 
Let $L_{n}=\boldsymbol{R}^{n} \times[0,1]^{\infty}$, namely

$$
L_{n}=\left\{\left(x_{1}, x_{2}, \ldots\right) ; 0 \leqq x_{k} \leqq 1 \text { for } k>n\right\} \subset \mathbb{R}^{\infty}
$$

On the set $L_{n}$, consider the product of $n$-dimensional Lebesgue measure and the uniform probability measures. Denote this measure with $m_{n}$.

The measure $m_{n}$ can be defined on $\mathbb{R}^{\infty}$ as follows:

$$
m_{n}(E)=m_{n}\left(E \cap L_{n}\right) \text { for measurable } E \subset \mathbb{R}^{\infty} .
$$

Evidently, $m_{n}$ is a $\sigma$-additive, $\sigma$-finite measure on $\mathbb{R}^{\infty}$.

Put

$$
\bar{\mu}=\sum_{n=1}^{\infty} \frac{1}{2^{n}} m_{n}
$$

$\bar{\mu}$ is $\sigma$-finite because $\bar{\mu}\left(\boldsymbol{R}^{\infty}-\bigcup_{n} L_{n}\right)=0$ and for $E \subset \mathbb{R}^{n}$ we have

$$
\begin{aligned}
\bar{\mu}\left(E \times[0,1]^{\infty}\right)= & \sum_{k=1}^{\infty} \frac{1}{2^{k}} m_{k}\left(E \times[0,1]^{\infty}\right) \\
& \leqq \text { Lebesgue measure of } E .
\end{aligned}
$$

Now, we shall consider the projection of $\bar{\mu}$ on $\mathbb{R}^{n}$. For a Borel subset $E$ of $\boldsymbol{R}^{n}$, we have

$$
p_{n}^{-1}(E) \cap L_{k}=\left\{\begin{array}{l}
E \times \boldsymbol{R}^{k-n} \times[0,1]^{\infty} \text { if } k>n \\
E \times[0,1]^{\infty} \text { if } k=n \\
E \cap\left(\boldsymbol{R}^{k} \times[0,1]^{n-k}\right) \times[0,1]^{\infty} \text { if } k<n .
\end{array}\right.
$$

Therefore if the Lebesgue measure of $E$ is zero, we have $m_{k}\left(p_{n}^{-1}(E)\right)$ $=0$ for any $k$ so that $\bar{\mu}\left(p_{n}^{-1}(E)\right)=0$. On the contrary, if the Lebesgue measure of $E$ is positive, $m_{k}\left(p_{n}^{-1}(E)\right)=\infty$ for $k>n$ so that $\bar{\mu}\left(p_{n}^{-1}(E)\right)=\infty$. Thus, the projection of $\bar{\mu}$ on $\mathbb{R}^{n}$ is just identical with (4.3).

We shall remark that the above $\bar{\mu}$ is far from translationally invariant measure, and in this sense it does not qualify as the $\infty$-dimensional Lebesgue measure.

\section{$\S 5$. Lorentz Invariant Measure}

Considering the projective limit of uniform measures on $n$-dimensional 
spheres, we obtain the infinite dimensional gaussian measure which is unique as the rotationally invariant one. (See [6] and [7]).

Similar discussions for the Lorentz invariant measure are the contents of this section.

1) Let $l(x)=-x_{0}^{2}+x_{1}^{2}+\cdots+x_{n}^{2}$ be the Lorentz metric on $\mathbb{R}^{n+1}$, and put

$$
\begin{aligned}
& H_{n}^{1}=\left\{x \in \mathbb{R}^{n+1} ; l(x)=1\right\} \\
& H_{n}^{2}=\left\{x \in \mathbb{R}^{n+1} ; l(x)=-1\right\}
\end{aligned}
$$

$H_{n}^{1}$ is a hyperboloid of one sheet, while $H_{n}^{2}$ is a pair of hyperboloids of two sheets.

Let $\mathfrak{R}_{n}$ be the $n$-dimensional Lorentz group, namely the group of linear operators on $\mathbb{R}^{n+1}$ which keep $l(x)$ invariant. It acts transitively on each of $H_{n}^{1}$ and $H_{n}^{2}$.

We shall define the measure $\mu_{n}$ on $H_{n}^{1}$ or $H_{n}^{2}$ as follows:

$$
d \mu_{n}=\frac{d \sigma}{r(x)}
$$

where $d \sigma$ is the area element and $r(x)=\left(x_{0}^{2}+x_{1}^{2}+\cdots+x_{n}^{2}\right)^{\frac{1}{2}} . \quad \mu_{n}$ is the unique (except a constant factor) measure which is $\mathbb{L}_{n}$-invariant and $\sigma$-finite on $H_{n}^{1}$ or $H_{n}^{2}$.

2) For a while, we shall discuss about $H_{n}^{1}$ only. Put

$$
\Omega_{n}=H_{n}^{1} \cup\left\{\omega_{n}\right\}
$$

where $\omega_{n}$ is a fictitious additional point. Putting $\mu_{n}\left(\left\{\omega_{n}\right\}\right)=\infty, \mu_{n}$ is regarded as a measure on $\Omega_{n}$.

For $m>n$, we shall define the projection $p_{m n}$ as follows :

$$
\begin{aligned}
& \left\{\begin{array}{l}
p_{m n}\left(\omega_{m}\right)=\omega_{n} \\
p_{m n}\left(x_{0}, x_{1}, \ldots, x_{m}\right)=\omega_{n} \quad \text { if } \quad r_{m n}^{2} \geqq 1 \\
p_{m n}\left(x_{0}, x_{1}, \ldots, x_{m}\right)
\end{array} \quad=\left(x_{0}, x_{1}, \ldots, x_{n}\right) / \sqrt{1-r_{m n}^{2}} \in H_{n}^{1} \quad \text { if } \quad r_{m n}^{2}<1,\right.
\end{aligned}
$$

where $r_{m n}^{2}=x_{n+1}^{2}+\cdots+x_{m}^{2}$. 
Then, we have for any $E \subset H_{n}^{1}$

$$
\begin{aligned}
\mu_{m}\left(p_{m n}^{-1}(E)\right) & =\mu_{n}(E) \times \int_{r_{m n}^{2}<1}\left(1-r_{m n}^{2}\right)^{\frac{n-1}{2}} d x_{n+1} \ldots d x_{m} \\
& =2^{m-n} \mu_{n}(E) \prod_{k=n}^{m-1} \int_{0}^{\frac{\pi}{2}} \cos ^{k} \theta d \theta \\
& =\pi^{\frac{m-n}{2}} \mu_{n}(E) \Gamma\left(\frac{n+1}{2}\right) / \Gamma\left(\frac{m+]}{2}\right) .
\end{aligned}
$$

Therefore, the family of measures $\left\{c_{n} \mu_{n}\right\}$ where

$$
c_{n}=\pi^{-\frac{n}{2}} \Gamma\left(\frac{n+1}{2}\right)
$$

is self-consistent.

Let the projective limit of $\Omega_{n}$ be $\Omega$. Since each $\Omega_{n}$ is the union of $H_{n}^{1}$, the $\sigma$-finite part, and $\left\{\omega_{n}\right\}$, the essentially infinite part, the family $\left\{c_{n} \mu_{n}\right\}$ satisfies the condition (2.8)' and can be extended to a $\sigma$-additive measure on $\Omega$. (The proof of this statement will be given in $\S 7$ ). The extension is unique on

$$
\Omega_{0}=\bigcup_{n} p_{n}^{-1}\left(H_{n}^{1}\right) \subset \Omega .
$$

We shall denote with $\mu$ this unique $\sigma$-additive measure on $\Omega_{0}$.

3) Using a similar discussion in [6] or [7], we shall identify $\mu$ with the measure $\exp \left(x_{0}^{2}\right) g_{1}$ on $\mathbb{R}^{\infty}$, where $g_{1}$ is the infinite dimensional gaussian measure with variance 1 .

For $\omega \in p_{n}^{-1}\left(H_{n}^{1}\right)$, let $x_{k}^{(n)}(\omega)$ be the $k$-th orthogonal coordinate of $p_{n}(\omega)$, namely

$$
p_{n}(\omega)=\left(x_{0}^{(n)}(\omega), \ldots, x_{n}^{(n)}(\omega)\right) \in H_{n}^{1} .
$$

First, we shall prove that for fixed $k$, an appropriate subsequence of $\left\{\sqrt{n} x_{k}^{(n)}(\omega)\right\}$ converges almost everywhere on $\Omega_{0}$. Since $\Omega_{0}=\bigcup_{j} \bigcup_{i} p_{j}^{-1}\left(E_{i j}\right)$, where $E_{i j} \subset H_{j}^{1}$ such that $\bigcup_{i} E_{i j}=H_{j}^{1}$, the almost everywhere convergence on each $p_{j}^{-1}\left(E_{i j}\right)$ implies the same on $\Omega_{0}$.

Now, we shall prove the restriction of $\left\{\sqrt{n} x_{k}^{(n)}(\omega)\right\}$ on $p_{j}^{-1}\left(E_{i j}\right)$ converges in $L^{2}$-sense, supposing that each $E_{i j}$ is a bounded set. For $m \geqq n$ 
$\geqq j \geqq k$, after some calculations we have

$$
\begin{aligned}
\int_{p_{m j}^{-1}\left(E_{i j}\right)} x_{k}^{(m)} x_{k}^{(n)} d\left(c_{m} \mu_{m}\right) \\
=\int_{E_{i j}} x_{k}^{(j) 2} d\left(c_{j} \mu_{j}\right) \times \frac{j+1}{2} \frac{\Gamma\left(\frac{n+2}{2}\right)}{\Gamma\left(\frac{n+3}{2}\right)} \frac{\Gamma\left(\frac{m+1}{2}\right)}{\Gamma\left(\frac{m+2}{2}\right)} .
\end{aligned}
$$

Since $\Gamma(t) / \Gamma\left(t+\frac{1}{2}\right) \sim 1^{\sqrt{ }} \bar{t}$ asymptotically, this shows that $\sqrt{n} x_{k}^{(n)}$ converges in $L^{2}$-sense on $p_{j}^{-1}\left(E_{i j}\right)$.

Now, put

$$
X_{k}(\omega)=\lim _{n} \sqrt{n} x_{k}^{(n)}(\omega)
$$

(Rigorously, the limit of an appropriate subsequence). Consider the mapping $\Phi: \Omega_{0} \ni \omega \rightarrow\left(X_{k}(\omega)\right) \in \boldsymbol{R}^{\infty}$. $\Phi$ is defined almost everywhere on $\Omega_{0}$.

Since $x_{k}^{(n)}=x_{k}^{(m)} / \sqrt{1-r_{m n}^{2}}=x_{k}^{(m)} / \sqrt{-x_{0}^{(m) 2}+x_{1}^{(m) 2}+\ldots+x_{n}^{(m) 2}}$, letting $m \rightarrow \infty$ we have

$$
x_{k}^{(n)}(\omega)=X_{k}(\omega) / \sqrt{-X_{0}^{2}(\omega)+X_{1}^{2}(\omega)+\ldots+X_{n}^{2}(\omega)} .
$$

This shows $\Phi$ is one-to-one.

By the mapping $\Phi$, the measure $\mu$ on $\Omega_{0}$ is mapped to a measure on $\boldsymbol{R}^{\infty}$, which is proved to be $2 \sqrt{\pi} \exp \left(X_{\mathbf{0}}^{\mathbf{2}}\right) g_{1}$. For this purpose, we shall calculate the characteristic function of $\exp \left(-X_{0}^{2}\right) \mu$.

$$
\begin{aligned}
& \int_{\Omega_{0}} \exp \left[i \sum_{k=0}^{K} \alpha_{k} X_{k}(\omega)-X_{0}^{2}(\omega)\right] d \mu(\omega) \\
& \quad=\lim _{j \rightarrow \infty} \lim _{i \rightarrow \infty} \lim _{n \rightarrow \infty} \int_{p_{n j}^{-1}\left(E_{i j}\right)} \exp \left[i \sqrt{n} \sum_{k=0}^{K} \alpha_{k} x_{k}^{(n)}-n x_{0}^{(n) 2}\right] d\left(c_{n} \mu_{n}\right) \\
& \quad=2 \sqrt{\pi} \exp \left[-\frac{1}{2} \sum_{k=0}^{K} \alpha_{k}^{2}\right] .
\end{aligned}
$$

(The detailed calculation is omitted here). This shows $\exp \left(-X_{0}^{\mathbf{2}}\right) \mu$ $=2 \sqrt{\pi} g_{1}$.

4) The measure $h=\exp \left(x_{0}^{2}\right) g_{1}$ can be constructed on a nuclear extension $L^{*}$ of a Hilbert space, and can be interpreted as a Lorentz invariant measure on $L^{*}$. 
Let $H$ be a real Hillert space, and $L$ be a locally convex space which is densely and nuclearly imbedded in $H$. Let $\left\{e_{0}, e_{1}, \ldots, e_{n}, \ldots\right\}$ be a C.O.N.S. of $H$ lying in $L$.

Consider the following operator $J$ on $H$ :

$$
\left\{\begin{array}{l}
\int e_{0}=-e_{0} \\
\int e_{k}=e_{k}
\end{array} \quad(k \geqq 1)\right.
$$

Let $\mathbb{R}_{\infty}$ be the Lorentz group of $H$, namely the group of such linear operators on $H$ that keep the Lorentz metric $\langle x, J x\rangle$ invariant. Let $\mathfrak{Q}_{L}$ be a subgroup of $\mathfrak{R}_{\infty}$ which consists of such $A$ that keep $L$ invariant and act homeomorphically on $L$. Then, identifying $A$ with $J^{*}\left(A^{-1}\right)^{*} J^{*}, \mathfrak{\Omega}_{L}$ can be regarded as a transformation group on $L^{*}$, the dual space of $L$. (c.f. $\S 3,3)$ ).

Since the gaussian measure $g_{1}$ can be defined on $L^{*}$, the measure $h=\exp \left(\left\langle x, e_{0}\right\rangle^{2}\right) g_{1}$ can be considered on $L^{*}$. This $h$ is $\mathfrak{L}_{L}$-invariant as shown below.

Let $\mathrm{R}$ be a finite dimensional subspace of $L$ including $e_{0}$. The projection of $h$ on $L^{*} / R^{\perp} \simeq R^{*}$ is in the form

$$
\exp \left[\frac{1}{2}\left(x_{0}^{2}-x_{1}^{2}-\ldots-x_{n}^{2}\right)\right] d x_{0} d x_{1} \ldots d x_{n}
$$

which is Lorentz invariant. This shows that $h$ itself is Lorentz invariant, because the extension to a $\sigma$-additive measure is unique.

5) Next, we shall discuss on $H_{n}^{2}$.

The measure $\mu_{n}$ defined in (5.3) is Lorentz invariant on $H_{n}^{2}$.

For $m>n$, we shall define the projection $p_{m n}: H_{m}^{2} \rightarrow H_{n}^{2}$ as follows:

$$
p_{m n}\left(x_{0}, x_{1}, \ldots, x_{m}\right)=\left(x_{0}, x_{1}, \ldots, x_{n}\right) / \sqrt{1+r_{m n}^{2}}
$$

where $r_{m n}^{2}=x_{n+1}^{2}+\ldots+x_{m}^{2}$.

Then, we have for any $E \subset H_{n}^{2}$,

$$
\begin{aligned}
\mu_{m}\left(p_{m n}^{-1}(E)\right) & =\mu_{n}(E) \times \int\left(1+r_{m n}^{2}\right)^{\frac{n-1}{2}} d x_{n+1} \ldots d x_{m} \\
& =2^{m-n} \mu_{n}(E) \times \prod_{k=n}^{m-1} \int_{0}^{\infty} \cosh ^{k} t d t
\end{aligned}
$$


Thus, the ratio of normalization constants $c_{n}$ is infinity (c.f. 2) in the case of $H_{n}^{1}$ ), and the family $\left\{\mu_{n}\right\}$ never becomes self-consistent by a multiplication of constant factors. Instead, if we consider the family of $(0, \infty)$-type measures, we can not determine the projective limit measure. This situation is just the same as the case of Lebesgue measures (c.f. $\S 4,5$ )).

D. Shale and W. F. Stinespring constructed a Lorentz invariant "integration" on the infinite dimensional hyperboloids of two sheets ([8]). But it is not a measure in an exact sense, but only a measure-like object. Its justification will be achieved if we construct the corresponding (in some sense) $\sigma$-additive measure. Unfortunately, our theory turnes out to be invalid for this purpose.

6) Finally, we shall construct a $\sigma$-finite measure whose projection on $H_{n}^{2}$ is $(0, \infty)$-type (c.f. $\S 4,6)$ ).

Let $M_{n}$ be a subset of $H_{n}^{2}$ that satisfies:

$$
\begin{array}{r}
\mu_{n}\left(p_{n, n-1}^{-1}(E) \cap M_{n}\right)=\mu_{n-1}(E) \\
\text { for any } E \subset H_{n-1}^{2} .
\end{array}
$$

For instance, it is sufficient to put

$$
M_{n}=\left\{\left(x_{0}, x_{1}, \ldots, x_{n}\right) \in H_{n}^{2} ; 0 \leqq x_{n} \leqq \sinh t_{n}\right\}
$$

where $\int_{0}^{t_{n}} \cosh ^{n-1} \tau d \tau=1$.

For these $\left\{M_{n}\right\}$, we shall put

$$
L_{n}=\bigcap_{k>n} p_{k}^{-1}\left(M_{k}\right) \subset \Omega .
$$

Since the family $\left\{\mu_{k}\right\}_{k \geqq n}$ is self-consistent on $L_{n}$, it determines a $\sigma$-additive measure $m_{n}$ on $L_{n}$. The measure $m_{n}$ can be defined on $\Omega$, putting

$$
m_{n}(E)=m_{n}\left(E \cap L_{n}\right) \text { for } E \subset \Omega .
$$

Now, consider the following measure $\bar{\mu}$ on $\Omega$ :

$$
\bar{\mu}=\sum_{n=1}^{\infty} \frac{1}{2^{n}} m_{n}
$$

Similar discussions in $\S 4,6)$ show that $\bar{\mu}$ is $\sigma$-finite, and its projection on 
each $H_{n}^{2}$ is $(0, \infty)$-type. Thus, $\bar{\mu}$ is the requested measure. However, $\bar{\mu}$ is far from Lorentz invariant measure.

\section{$\S 6 . \quad$ Non $\sigma$-finite Case}

In this section, we shall return to the general discussion on the extendability for infinite measures.

1) Let $\left\{\mu_{n}\right\}$ be a self-consistent family of infinite measures defined on measurable spaces $\left\{\Omega_{n}, \mathfrak{B}_{n}\right\}$ which allow a unique extension for probability measures. Let $\{\Omega, \mathfrak{B}\}$ be the projective limit measurable space of $\left\{\Omega_{n}, \mathfrak{B}_{n}\right\}$.

First, we shall resume the results in $\S 2,3) \sim 5$ ). If the family $\left\{\mu_{n}\right\}$ is extended to a $\sigma$-additive measure $\bar{\mu}$ on $\mathfrak{B}$, we must have

$$
\bar{\mu}(E \cap B)=\mu^{(B)}(E) \quad \text { for } \quad B \in \mathfrak{H}_{0}, E \in \mathfrak{B} \text {, }
$$

where $\mathfrak{B}_{0}$ and $\mu^{(B)}$ are defined in $\left.\S 2,3\right)$. Especially if $E \cap B \in \mathfrak{F}=$ $\bigcup_{n} p_{n}^{-1}\left(\mathfrak{B}_{n}\right)$, we must have

$$
\mu(E \cap B)=\mu^{(B)}(E),
$$

where $\mu$ is the finitely additive measure on $\mathfrak{F}$ defined by $\left\{\mu_{n}\right\}$.

The condition (2.8) is equivalent to

$$
\mu(E)=\sum_{n=1}^{\infty} \mu\left(E \cap A_{n}\right) \quad \text { for } \quad E \in \mathfrak{F} \cap \mathfrak{B}_{0},
$$

where $\left\{A_{n}\right\}$ is such that $A_{n} \in \mathfrak{F}, \mu\left(A_{n}\right)<\infty$ and $E \subset \bigcup_{n=1}^{\infty} A_{n} . \quad(2.8)^{\prime}$ is also equivalent to the following condition $(\mathbb{E G})$ :

(EG) If $E \in \mathfrak{F} \cap \mathfrak{B}_{0}, \mu(E)=\infty$, then for any $N>0$ there exists such $F \in \mathfrak{F}$ that satisfies $F \subset E$ and $N<\mu(F)<\infty$.

Therefore, $(2.8)$ or $(2.8)^{\prime}$ or $(\mathbf{E G})$ is a necessary condition for the extendability of $\left\{\mu_{n}\right\}$.

2) From (2.5) we have

$$
\bar{\mu}(B)=\mu^{(B)}(B) \text { for } \quad B \in \mathfrak{B}_{0} .
$$

So, the extension (if possible) must be unique on $\mathfrak{B}_{0}$. Especially if 
$\Omega \in \mathfrak{B}_{0}$, then we have $\mathfrak{B}_{0}=\mathfrak{B}$ and the extension is unique on $\mathfrak{B}$. A special case is the $\sigma$-finite case as stated in Theorem 1 in $\S 2,4$ ).

Now, we shall prove that the condition (EG) is sufficient for the extendability. For this purpose, we shall define $\bar{\mu}$ by (6.1) on $\mathfrak{B}_{0}$, and on $\mathfrak{B}-\mathfrak{B}_{0}$ we shall put

$$
\bar{\mu}(E)=\infty \quad \text { if } \quad E \in \mathfrak{B}-\mathfrak{B}_{0} .
$$

This $\bar{\mu}$ is $\sigma$-additive, because for any sequence $\left\{E_{n}\right\}$ of mutually disjoint measurable sets,

$$
\bar{\mu}\left(\bigcup_{n} E_{n}\right)=\sum_{n} \bar{\mu}\left(E_{n}\right)
$$

can be checked as below.

If some $E_{n} \notin \mathfrak{B}_{0}$, then evidently $\cup_{n} E_{n} \notin \mathfrak{B}_{0}$ so that the both hand sides of (6.3) are infinity. If each $E_{n} \in \mathfrak{B}_{0}$, then $E=\bigcup_{n} E_{n} \in \mathfrak{B}_{0}$ and we have

$$
\begin{aligned}
\bar{\mu}(E)=\mu^{(E)}(E) & =\sum_{n=1}^{\infty} \mu^{(E)}\left(E_{n}\right) \\
& =\sum_{n=1}^{\infty} \mu^{\left(E_{n}\right)}\left(E_{n}\right)=\sum_{n=1}^{\infty} \bar{\mu}\left(E_{n}\right),
\end{aligned}
$$

using the definition of $\bar{\mu}, \sigma$-additivity of $\mu^{(E)}$, and the relation (2.4).

Next, $\bar{\mu}$ is an extension of $\left\{\mu_{n}\right\}$. To show this, it is sufficient to prove $\bar{\mu}(E)=\mu(E)$ for $E \in \mathfrak{F}$. If $E \in \mathfrak{F} \cap \mathfrak{B}_{0}$, then from the definition (2.3) of $\mu^{(E)}$ we have

$$
\bar{\mu}(E)=\mu^{(E)}(E)=\sum_{n=1}^{\infty} \mu^{\left(A_{n}\right)}(E)=\sum_{n=1}^{\infty} \mu\left(A_{n} \cap E\right) .
$$

So under the condition $(2.8)^{\prime}$ we have $\bar{\mu}(E)=\mu(E)$. If $E \in \mathfrak{F}-\mathfrak{B}_{0}$, we have $\mu(E)=\infty$ from the definition of $\mathfrak{B}_{0}$, so that $\bar{\mu}(E)=\mu(E)=\infty$.

Since the extension is unique on $\mathfrak{B}_{0}$ and we put $\bar{\mu}$ always infinity outside $\mathfrak{B}_{0}$, this $\bar{\mu}$ is evidently the possible largest extension. Hereafter, we shall denote with $\tilde{\mu}$ this largest extension.

Theorem 2. A self-consistent family $\left\{\mu_{n}\right\}$ of infinite measures is extendable to a $\sigma$-additive measure on $\mathfrak{B}$, if and only if the condition $(\mathbb{E C})$ 
(or equivalently the condition (2.8)') is satisfied.

Under the condition $(\mathbb{E C})$, the extension is unique on $\mathfrak{B}_{0}$, It is not necessarily unique on $\mathfrak{B}$, but there exists the largest extension $\tilde{\mu}$.

Remark that if $\Omega \notin \mathfrak{B}_{0} . \tilde{\mu}$ is not $\sigma$-finite. If each $\mu_{n}$ is $(0, \infty)$-type (or essentially infinite), then $\tilde{\mu}$ is also $(0, \infty)$-type (or essentially infinite).

3) The following condition $(\mathbb{F G})$ is stronger than $(\mathbb{E} G)$ :

$(\mathbf{F G})$ If $E \in \mathfrak{F}, \mu(E)=\infty$, then for any $N>0$ there exists such $F \in \mathfrak{F}$ that satisfies $F \subset E$ and $N<\mu(F)<\infty$.

Under the condition $(\mathbf{F G})$, we shall construct the smallest extension $\stackrel{\sim}{\sim}$ of $\left\{\mu_{n}\right\}$.

Put

$$
\underset{\sim}{\mu}(E)=\sup _{\substack{B \subset E \\ B \in \mathfrak{B}_{0}}} \mu^{(B)}(B) \quad \text { for } \quad E \in \mathfrak{B} .
$$

Especially if $E \in \mathfrak{B}_{0}$ we have $\underset{\sim}{\mu}(E)=\mu^{(E)}(E)$.

First, we shall prove that $\underset{\sim}{\mu}$ is a $\sigma$-additive measure. Let $\left\{E_{n}\right\}$ be a sequence of mutually disjoint measurable sets. For any $B \subset E=\cup_{n} E_{n}$ we have $B=\bigcup_{n}\left(B \cap E_{n}\right)$, hence

$$
\mu^{(B)}(B)=\sum_{n} \mu^{(B)}\left(B \cap E_{n}\right)=\sum_{n} \mu^{\left(B \cap E_{n}\right)}\left(B \cap E_{n}\right) \leqq \sum_{n} \underset{\sim}{\mu}\left(E_{n}\right)
$$

so that we have $\underset{\sim}{\mu}(E) \leqq \sum_{n} \underset{\sim}{\mu}\left(E_{n}\right)$. Conversely, for any $B_{n} \subset E_{n}$ we have $\bigcup_{n} B_{n} \subset E$, hence

$$
\sum_{n} \mu^{\left(B_{n}\right)}\left(B_{n}\right)=\sum_{n} \mu^{\left(\cup B_{n}\right)}\left(B_{n}\right)=\mu^{\left(\cup B_{n}\right)}\left(\cup B_{n}\right) \leqq \underset{\sim}{\mu}(E)
$$

so that we have $\sum_{n} \underset{\sim}{\mu}\left(E_{n}\right) \leqq \underset{\sim}{\mu}(E)$.

Next, $\underset{\sim}{\mu}$ is an extension of $\left\{\mu_{n}\right\}$. Because, if $E \in \mathfrak{F}, \mu(E)<\infty$, we have $E \in \mathfrak{B}_{0}$ and $\underset{\sim}{\mu}(E)=\mu^{(E)}(E)=\mu(E)$. If $E \in \mathfrak{F}, \mu(E)=\infty$, the condition ( $\mathbb{F G}$ ) assures

$$
\underset{\sim}{\mu}(E) \geqq \mu^{(F)}(F)=\mu(F)>N
$$

so that $\underset{\sim}{\mu}(E)=\infty$. Thus we get:

Theorem 3 Under the condition $(\boldsymbol{F C})$, there exists the smallest extension $\underset{\sim}{\mu}$. 
Remark that $(\mathbf{F G})$ is also necessary in order that the measure $\underset{\sim}{\mu}$ defined in (6.4) is an extension of $\left\{\mu_{n}\right\}$. If $E \in \mathfrak{F}, \mu(E)=\infty$ implies $\underset{\sim}{\mu}(E)=\infty$, then there exists $B \in \mathfrak{B}_{0}, B \subset E$ such that $\mu^{(B)}(B)>2 N$. Suppose that $B \subset \bigcup_{n} A_{n}, A_{n} \in \mathfrak{F}$ and $\mu\left(A_{n}\right)<\infty$. Since

$$
\mu^{(B)}(B)=\sum_{n=1}^{\infty} \mu^{\left(A_{n}\right)}(B) \leqq \sum_{n=1}^{\infty} \mu^{\left(A_{n}\right)}(E)=\sum_{n=1}^{\infty} \mu\left(E \cap A_{n}\right),
$$

we have for some $k$

$$
N<\sum_{n=1}^{k} \mu\left(E \cap A_{n}\right)=\mu\left(\bigcup_{n=1}^{k}\left(E \cap A_{n}\right)\right)
$$

Evidently $F=\bigcup_{n=1}^{k}\left(E \cap A_{n}\right)$ satisfies the request of $(\mathbf{F G})$.

4) If the condition (FG) is not fulfilled, the smallest extension may or may not exist. Even if it exists, it takes more complicated expression than (6.4), but we shall not enter this problem. We shall be contented only with examples which have no smallest extensions.

In $\S 4,3$ ), we considered a measure $\bar{\mu}$ on $\mathbb{Z}_{0}^{\infty}$ whose projection on $\mathbb{Z}^{n}$ is essentially infinite. Since $\mathbb{Z}_{0}^{\infty}$ is a countable set, we can affix a numerical order to its elements;

$$
\mathbb{Z}_{0}^{\infty}=\left\{x_{1}, x_{2}, x_{3}, \ldots\right\}
$$

Consider the measure $\bar{\mu}_{k}(E)=\bar{\mu}\left(E-\left\{x_{k}\right\}\right)$. For any $k$, the projection of $\bar{\mu}_{k}$ on $\mathbb{Z}^{n}$ is essentially infinite. In other words, $\bar{\mu}_{k}$ is an extension of the family of essentially infinite measures. If the smallest extension would exist, $\underset{\sim}{\mu} \leqq \bar{\mu}_{k}$ for any $k$ implies $\underset{\sim}{\mu}\left(\left\{x_{k}\right\}\right)=0$ for any $k$ so that $\underset{\sim}{\mu} \equiv 0$. Since the zero measure is not an extension of the family of essentially infinite measures, this shows the non-existence of the smallest extension.

Similarly, consider a measure $\bar{\mu}$ on $\mathbb{R}^{\infty}$ defined in $\left.\S 4,6\right)$. This $\bar{\mu}$ is an extension of $(0, \infty)$-type measures on $\mathbb{R}^{n}$. Put $\bar{\mu}_{k}(E)=\bar{\mu}\left(E-L_{k}\right)$, where $L_{k}$ is given in (4.4). Then for any $k, \bar{\mu}_{k}$ is an extension of $(0, \infty)$ type measures on $\mathbb{R}^{n}$, but if $\underset{\sim}{\mu} \leqq \bar{\mu}_{k}$ for any $k$, we have $\underset{\sim}{\mu} \equiv 0$. Therefore, in this case also, the smallest extension does not exist. Similar discussions show that the family of $(0, \infty)$-type measures on $H_{n}^{2}$ (c.f. $\left.\S 5,6\right)$ ) does not have the smallest extension. 


\section{$\S 7 . \quad \sigma$-finite Plus Essentially Infinite Case}

In this section, we shall give a sufficient condition for the extendability condition $(\mathbf{E G})$, and as an application we shall treat the infinite product of finite- or countable-point sets. The latter is closely related with the symbolic representation of flows, as explained in $\S 8$.

1) The following proposition is fundamental in this section.

Proposition 7.1. Let $\left\{\Omega_{n}, \mathfrak{B}_{n}, \mu_{n}\right\}$ be a self-consistent family of infinite measures, and suppose that each $\Omega_{n}$ can be decomposed into the union of $\sigma$-finite part and essentially infinite part; namely

$$
\begin{aligned}
& \Omega_{n}=\Omega_{n}^{1} \cup \Omega_{n}^{2}, \Omega_{n}^{1} \in \mathfrak{B}_{n}, \Omega_{n}^{2} \in \mathfrak{B}_{n}, \\
& \mu_{n} \mid \Omega_{n}^{1} \text { is } \sigma \text {-finite, } \\
& \mu_{n} \mid \Omega_{n}^{2} \text { is essentially infinite. }
\end{aligned}
$$

Then, the family of measures $\left\{\mu_{n}\right\}$ satisfies the extendability condition $(\boldsymbol{E} C)$ in $\S 6, \mathbf{1})$.

Remark Here, we assume that any one-point set belongs to $\mathfrak{B}_{n}$.

Definition of essentially infiniteness is given in $§ 4,2)$.

Proof. We shall check the condition $(\mathbb{E C})$. Assume that $E \in \mathfrak{F} \cap \mathfrak{B}_{0}$ and $\mu(E)=\infty$. ( $\mu$ denotes the finitely additive measure on $\mathfrak{F}$ defined by $\left.\left\{\mu_{n}\right\}\right)$.

From the self-consistency condition of $\left\{\mu_{n}\right\}$, we know that $\left\{p_{n}^{-1}\left(\Omega_{n}^{1}\right)\right\}$ is an increasing sequence. It is easily seen that $E \in \mathfrak{B}_{0}$ is equivalent to $E \subset \bigcup_{n=1}^{\infty} p_{n}^{-1}\left(\Omega_{n}^{1}\right)$, namely to

$$
E \cap\left(\bigcap_{n=1}^{\infty} p_{n}^{-1}\left(\Omega_{n}^{2}\right)\right)=\phi .
$$

On the other hand, $E \in \mathfrak{F}=\bigcup_{m=1}^{\infty} p_{m}^{-1}\left(\mathfrak{B}_{m}\right)$ implies $E \in p_{m}^{-1}\left(\mathfrak{B}_{m}\right)$ for some $m$, and $\mu(E)=\mu_{m}\left(p_{m}(E)\right)$. If $E \subset p_{m}^{-1}\left(\Omega_{m}^{1}\right)$, then $p_{m}(E) \subset \Omega_{m}^{1}$ and the $\sigma$-finiteness of $\mu_{m}$ on $\Omega_{m}^{1}$ assures the existence of $F \in \mathfrak{F}$ requested in the condition (EG).

If $E \in p_{m}^{-1}\left(\mathfrak{B}_{m}\right)$ and $E \not \subset p_{m}^{-1}\left(\Omega_{m}^{1}\right)$, suppose that $x \in E \cap p_{m}^{-1}\left(\Omega_{m}^{2}\right)$ and 
put $x_{m}=p_{m}(x)$. Since $x_{m} \in \Omega_{m}^{2}$, we have $\mu_{m}\left(\left\{x_{m}\right\}\right)=\infty$, therefore from the self-consistency condition of $\left\{\mu_{n}\right\}$, we have $\mu_{m+1}\left(p_{m+1}^{-1}, m\left(\left\{x_{m}\right\}\right)\right)=\infty$.

If $p_{m+1, m}^{-1}\left(\left\{x_{m}\right\}\right) \subset \Omega_{m+1}^{1}$, then the condition $(\mathbf{E C})$ is satisfied for $E$ in virtue of $\sigma$-finiteness of $\mu_{m+1}$ on $\Omega_{m+1}^{1}$. If $p_{m+1, m}^{-1}\left(\left\{x_{m}\right\}\right) \not \subset \Omega_{m+1}^{1}$, choose a point

$$
x_{m+1} \in p_{m+1, m}^{-1}\left(\left\{x_{m}\right\}\right) \cap \Omega_{m+1}^{2} .
$$

Repeat the similar discussion for $p_{m+2, m+1}^{-1}\left(\left\{x_{m+1}\right\}\right)$. If it is contained in $\Omega_{m+2}^{1}$, the condition $(\mathbf{E} \mathbf{C})$ is satisfied for $E$. If it intersects with $\Omega_{m+2}^{2}$, choose a point $x_{m+2}$ as above.

Repeating these procedures, if $E$ does not satisfy (EC), we can get a sequence $x_{m}, x_{m+1}, x_{m+2}, \ldots$ such that $x_{n} \in \Omega_{n}^{2}$ and $p_{n+1, n}\left(x_{n+1}\right)=x_{n}$ for $n \geqq m$. Therefore, from the definition of projective limit space $(\S 1)$, the sequence $x^{\prime}=\left(x_{m}, x_{m+1}, x_{m+2}, \ldots\right)$ belongs to $\Omega$.

Since $p_{m}\left(x^{\prime}\right)=x_{m}=p_{m}(x) \in p_{m}(E)$ and $E \in p_{m}^{-1}\left(\mathfrak{B}_{m}\right)$, we have $x^{\prime} \in E$. On the other hand, $p_{n}\left(x^{\prime}\right)=x_{n} \in \Omega_{n}^{2}$ implies $x^{\prime} \in p_{n}^{-1}\left(\Omega_{n}^{2}\right)$ for any $n \geqq m$. Thus, we get the negation of (7.2), hence $E \notin \mathfrak{B}_{0}$. In other words, any $E \in \mathfrak{F} \cap \mathfrak{B}_{0}$ must satisfy the condition $(\mathbf{E G})$.

(q.e.d.)

Remark. In Prop. 7.1, even if some (or all) $\mu_{n} \mid \Omega_{n}^{1}$ is finite (instead of $\sigma$-finite), the proposition is evidently true.

Corollary. If each $\Omega_{n}$ consists of finite or countable points, then any self-consistent family of measures $\left\{\Omega_{n}, \mathfrak{B}_{n}, \mu_{n}\right\}$ is extendable.

Because, in this case, $\Omega_{n}$ can necessarily be decomposed as in Prop. 7.1, namely:

$$
\left\{\begin{array}{l}
\Omega_{n}^{1}=\left\{x \in \Omega_{n} ; \mu_{n}(\{x\})<\infty\right\}, \\
\Omega_{n}^{2}=\left\{x \in \Omega_{n} ; \mu_{n}(\{x\})=\infty\right\} .
\end{array}\right.
$$

2) Let $I=\{1,2, \ldots, s\}$ be an $s$-point set, and put $\Omega_{n}=I^{n}$. Suppose that $\mathfrak{B}_{n}$ consists of all subsets of $\Omega_{n}$. A measure $\mu_{n}$ on $\mathfrak{B}_{n}$ is determined by a family of non-negative numbers $\left\{m_{k_{1} k_{2} \cdots k_{n}}\right\}$ where

$$
\begin{gathered}
m_{k_{1} k_{2}, \ldots k_{n}}=\mu_{n}\left(\left\{k_{1}, k_{2}, \ldots, k_{n}\right\}\right) \\
1 \leqq k_{1}, k_{2}, \ldots, k_{n} \leqq s
\end{gathered}
$$


The self-consistency condition is written as follows:

$$
\sum_{k=1}^{s} m_{k_{1} k_{2} \cdots k_{n} k}=m_{k_{1} k_{2} \cdots k_{n}}
$$

We shall assume that

$$
m_{k_{1} k_{2} \cdots k_{n}}\left\{\begin{array}{lll}
=\infty & \text { if } & k_{1}=k_{2}=\ldots=k_{n}=1 \\
<\infty & \text { otherwise. }
\end{array}\right.
$$

In other words, we assume

$$
\left\{\begin{array}{l}
\Omega_{n}^{1}=\Omega_{n}-\{1,1, \ldots, 1\} \\
\Omega_{n}^{2}=\{1,1, \ldots, 1\}
\end{array}\right.
$$

where $\Omega_{n}^{1}$ and $\Omega_{n}^{2}$ are defined in (7.3).

As proved in $\mathbb{1}$ ), the family of measures determined by (7.4) with the condition (7.5) is extendable to a $\sigma$-additive measure on $\{\Omega, \mathfrak{B}\}$, the projective limit measurable space of $\left\{\Omega_{n}, \mathfrak{B}_{n}\right\}$. The extension is unique on $\Omega_{0}=\bigcup_{n} p_{n}^{-1}\left(\Omega_{n}^{1}\right)$. Under the assumption $(7.6)^{\prime}$, we have

$$
\Omega_{0}=\Omega-\{1,1, \ldots\},
$$

so that the measure $\mu$ on $\Omega$ is determined except the value of $c=\mu(\{1,1, \ldots\})$. Putting $c=\infty$, we get the largest extension $\tilde{\mu}$.

The condition ( $\mathbf{F G}$ ) is fulfilled if and only if

$$
\lim _{n \rightarrow \infty} \mu_{n}\left(\Omega_{n}^{1}\right)=\infty
$$

under the assumption (7.6)'. If $F \in \mathfrak{F}$ and $\mu(F)<\infty$, we have $F \subset p_{m}^{-1}\left(\Omega_{m}^{1}\right)$ for some $m$, so that $\mu(F) \leqq \lim _{n \rightarrow \infty} \mu_{n}\left(\Omega_{n}^{1}\right)$. Therefore if (7.7) does not hold, the entire space $\Omega$ does not contain such $F \in \mathfrak{F}$ that satisfies

$$
\lim _{n \rightarrow \infty} \mu_{n}\left(\Omega_{n}^{1}\right)<\mu(F)<\infty
$$

Conversely, suppose that $E \in \mathfrak{F}$ and $\mu(E)=\infty$. If $E=p_{m}^{-1}\left(E_{m}\right)$ for some $E_{m} \in \mathfrak{B}_{m}$, then $E_{m} \cap \Omega_{m}^{2} \neq \phi$. Under the assumption (7.6)', this implies $\{1,1, \ldots, 1\} \in E_{m}$, therefore

$$
E \supset p_{m}^{-1}(\{1,1, \ldots, 1\}) \text {. }
$$


On the other hand, the set

$$
F_{n}=p_{m}^{-1}(\{1,1, \ldots, 1\}) \cap p_{n}^{-1}\left(\Omega_{n}^{1}\right)
$$

belongs to $\mathfrak{F}$ and we have

$$
\mu_{n}\left(\Omega_{n}^{1}\right)-\mu_{m}\left(\Omega_{m}^{1}\right)=\mu\left(F_{n}\right)<\mu_{n}\left(\Omega_{n}^{1}\right)<\infty
$$

Thus, letting $n \rightarrow \infty$, the condition (7.7) assures the condition (FG).

Remark that (7.7) is equivalent to

$$
\lim _{n \rightarrow \infty} \sum_{k_{1}, k_{2}, \ldots, k_{n}} m_{k_{1} k_{2} \cdots k_{n}}=\infty
$$

where $\Sigma^{\prime}$ means the summation except $k_{1}=k_{2}=\ldots=k_{n}=1$.

Now, if (7.7) is fulfilled, the condition (FG) being satisfied, the smallest extension exists and is given as (6.4). We can easily see that $\underset{\sim}{\mu}(\{1,1, \ldots\})$ $=0$. Furthermore, we can show that for any $c(0 \leqq c \leqq \infty)$, putting

$$
c=\mu(\{1,1, \ldots\})
$$

we get an extension of measures (7.4).

On the contrary, if (7.7) is not fulfilled, $c=\infty$ is the only possible choice and the extension is unique, because for any extension $\bar{\mu}$ we must have

$$
\begin{aligned}
\infty & =\bar{\mu}(\Omega)=\bar{\mu}(\Omega-\{1,1, \ldots\})+\bar{\mu}(\{1,1, \ldots\}) \\
& =\lim _{n \rightarrow \infty} \mu_{n}\left(\Omega_{n}^{1}\right)+\bar{\mu}(\{1,1, \ldots\}) .
\end{aligned}
$$

Thus, we obtained:

Proposition 7.2. Any self-consistent family of measures (7.4) on $I^{n}$ can be extended to a $\sigma$-additive measure on $\{\Omega, \mathfrak{B}\}$. Under the assumption (7.6), the extension is unique on $\Omega-\{1,1, \ldots\}$ and

(1) if (7.7) is satisfied, $\mu(\{1,1, \ldots\})$ can be chosen to be any non-negative value (including infinity), so that there exist continuously many extensions, (2) if (7.7) is not satisfied, $\mu(\{1,1, \ldots\})=\infty$ is the only possible choice and the extension is unique.

(3) The Prop. 7.2 is kept valid even if $\Omega_{n}$ is not the product space of 
the same set $I$.

Actually, Prop. 7.2 covers the following situation: For each n, $\Omega_{n}$ consists of finite elements, $\mathfrak{B}_{n}$ consists of all subsets of $\Omega_{n}$, and the value of $\mu_{n}$ is not infinity except one element (which is identified as $\{1,1, \ldots, 1\}$ in the discussions in 2 ), and the projective limit of which is identified as $\{1,1, \ldots\}$ in Prop. 7.2).

Prop. 7.2 for this situation is proved with slight modifications of the discussions in 2 ), and we do not repeat them.

Now, suppose that each $\Omega_{n}$ consists of countable elements. If $\Omega_{n}=\mathbb{N}^{n}$ where $N=\{1,2, \ldots\}$, the self-consistency condition is written as

$$
\sum_{k=1}^{\infty} m_{k_{1} k_{2}} \cdots k_{n} k=m_{k_{1} k_{2} \cdots k_{n}}
$$

Even if $\Omega_{n}$ is not the product space, the self-consistency of $\left\{\mu_{n}\right\}$ is written in a similar expression as $(7.5)^{\prime}$ for the values $\{m\}$.

An essential difference to the discussions in 2) appears in the condition (7.7). If $\Omega_{n}^{1}$ consists of countable elements, $\mu_{n}\left(\Omega_{n}^{1}\right)$ may be infinite and the discussion below (7.7) becomes invalid. However, for countable case also, if we replace (7.7) by

$$
\lim _{n \rightarrow \infty} \mu\left(p_{n}^{-1}\left(\Omega_{n}^{1}\right)-p_{m}^{-1}\left(\Omega_{m}^{1}\right)\right)=\infty \text { for any } m,
$$

the result is kept valid (though the proof requires some modification). If $\Omega_{n}=N^{n}$, the condition (7.8) is equivalent to

$$
\lim _{n \rightarrow \infty} \sum_{k_{m+1}, \ldots, k_{n}}^{\prime} m_{11 \cdots 1 k_{m+1} k_{m+2} \cdots k_{n}}=\infty
$$

where $\Sigma^{\prime}$ means the summation except $k_{m+1}=k_{m+2}=\ldots=k_{n}=1$.

Also the Prop. 7.2 is valid even if $\Omega_{n}$ consists of countable elements, if we replace the condition (7.7) by (7.8).

4) One more step for the further generalization is as follows:

Let $\left\{\Omega_{n}, \mathfrak{B}_{n}, \mu_{n}\right\}$ be a self-consistent family of infinite measures, and suppose that each $\Omega_{n}$ can be decomposed into the union of $\sigma$-finite part $\Omega_{n}^{1}$ and the essentially infinite part $\Omega_{n}^{2}$. As proved in $\left.\mathbb{1}\right),\left\{\mu_{n}\right\}$ can be extended to a $\sigma$-additive measure $\bar{\mu}$ on $\{\Omega, \mathfrak{B}\}$, the projective limit measurable space of $\left\{\Omega_{n}, \mathfrak{B}_{n}\right\}$. Since the extension is unique on $\Omega_{0}=\cup_{n} p_{n}^{-1}\left(\Omega_{n}^{1}\right)$, 
if we determine the value of $\bar{\mu}$ on $\bigcap_{n} p_{n}^{-1}\left(\Omega_{n}^{2}\right)$, then the measure $\bar{\mu}$ is determined on $\Omega$.

The following results are obtained easily:

\section{Proposition 7.3.}

(a) Under the condition

$$
\begin{aligned}
& \lim _{n \rightarrow \infty} \mu\left(p_{n}^{-1}\left(\Omega_{n}^{1}\right) \cap p_{m}^{-1}\left(\left\{x_{m}\right\}\right)\right)=\infty \\
& \quad \text { for any } m \text { and any } x_{m} \in \Omega_{m}^{2},
\end{aligned}
$$

any measure on $\bigcap_{n} p_{n}^{-1}\left(\Omega_{n}^{2}\right)$ gives an extension of $\left\{\mu_{n}\right\}$.

(b) Under the condition

$$
\begin{aligned}
& \lim _{n \rightarrow \infty} \mu\left(p_{n}^{-1}\left(\Omega_{n}^{1}\right)-p_{m}^{-1}\left(\Omega_{m}^{1}\right)\right)<\infty \\
& \text { for some } m,
\end{aligned}
$$

a measure $\bar{\mu}^{\prime}$ on $\cap p_{n}^{-1}\left(\Omega_{n}^{2}\right)$ gives an extension of $\left\{\mu_{n}\right\}$ on $\Omega$ if and only if $\bar{\mu}^{\prime}$ is an extension of essentially infinite measures on $\left\{\Omega_{n}^{2}, \mathfrak{B}_{n} \cap \Omega_{n}^{2}\right\}$.

If each $\Omega_{n}^{2}$ consists of one point, the condition (7.9) is just the negation of (7.10). In this case, the results of Prop. 7.3 are same with those of Prop. 7.2 ; namely under $(7.9) \bar{\mu}(\{1,1, \ldots\})$ can be any value and under (7.10) it must be infinity.

If $\Omega_{n}^{2}$ consists of more than one point, there exists the case which does not satisfy (7.9) nor (7.10). For such cases, the discussions on the abundance of extensions may be very complicated, and here we shall not study it.

\section{§ 8. Symbolic Representation of Flows}

Let $\{X, \mathfrak{B}, \mu\}$ be a measure space, and $\left\{T_{t}\right\}$ be a flow, namely a one-parameter group of measurable transformations on $X$. Assume the existence of finite generators $\left\{E_{k}\right\}_{1 \leqq k \leqq s}$, namely assume that $\left\{E_{k}\right\}$ is a finite partition of $X$ and $\mathfrak{B}$ is generated by

$$
\left\{T_{t} E_{k} ; t \in \boldsymbol{R}, 1 \leqq k \leqq s\right\}
$$

For any finite set of $t$-values $\left(t_{1}, t_{2}, \ldots, t_{n}\right)$, we put 


$$
m_{k_{1} k_{2} \cdots k_{n}}^{\left(t_{1} t_{2} \cdots t_{n}\right)}=\mu\left(\bigcap_{j=1}^{n} T_{t_{j}} E_{k_{j}}\right), \quad 1 \leqq k_{j} \leqq s
$$

Evidently, it fulfills the self-consistency condition:

$$
m_{k_{1} k_{2} \cdots k_{n}}^{\left(t_{1} t_{2} \cdots t_{n}\right)}=\sum_{k=1}^{s} m_{k_{1} k_{2} \cdots k_{n} k}^{\left(t_{1} t_{2} \cdots t_{n} t\right)} \quad \text { for any } t
$$

Even if $\left\{E_{k}\right\}$ is a countable (instead of finite) partition of $X$, the same relation holds if we replace $\sum_{k=1}^{s}$ by $\sum_{k=1}^{\infty}$.

Now suppose that $\mu$ is a probability measure on $X$. Then, $\left\{m_{k_{1} k_{2} \cdots k_{n}}^{\left(l_{1}, t_{2} \cdots t_{n}\right)}\right\}$ in (8.1) gives a self-consistent family of probability measures on $I^{R}$, where $I=\{1,2, \ldots, s\}$ (or if $\left\{E_{k}\right\}$ is countable, $I=\{1,2, \ldots\}=N$ ).

Let $p_{t}$ be the projection of $I^{\boldsymbol{R}}$ onto $I$ :

$$
p_{t_{0}}:(k(t)) \in I^{R} \longrightarrow k\left(t_{0}\right) \in I
$$

Consider the smallest $\sigma$-ring $\tilde{\mathfrak{B}}$ on $I^{\boldsymbol{R}}$ which makes all projections $p_{t}$ measurable. Then, Kolmogorov's extension theorem shows us that the family $\left\{m_{k_{1} k_{2} \cdots k_{n}}^{\left(t_{1} t_{2} \cdots t_{n}\right)}\right\}$ can be extended to the unique $\sigma$-additive measure $m$ on $\tilde{\mathfrak{B}}$.

Since the mapping $\Phi$ :

$$
\Phi: \bigcap_{j=1}^{n} p_{t_{j}}^{-1}\left(\left\{k_{j}\right\}\right) \in \tilde{\mathfrak{B}} \longrightarrow \bigcap_{j=1}^{n} T_{t_{j}} E_{k_{j}} \in \mathfrak{B}
$$

can be extended to a measure-preserving mapping from $\tilde{\mathfrak{B}}$ onto $\mathfrak{B}$, we can regard $\Phi$ as an isomorphism between two measure spaces $\{X, \mathfrak{B}, \mu\}$ and $\left\{I^{\boldsymbol{R}}, \tilde{\mathfrak{B}}, \boldsymbol{m}\right\}$. (However, $\Phi$ is not one-to-one from $\tilde{\mathfrak{B}}$ onto $\mathfrak{B}$. It is oneto-one modulo null-set).

From (8.3) we know that by the isomorphism $\Phi$, the transformation $T_{t}$ on $X$ is mapped to the shift operator $S_{t}$ on $I^{R}$ :

$$
S_{t_{0}}: k(t) \longrightarrow k\left(t+t_{0}\right)
$$

Thus we have the following conclusion:

Let $\{X, \mathfrak{B}, \mu\}$ be a probability measure space, and $\left\{T_{t}\right\}$ be a flow on $X$. Under the assumption of the existence of finite (or countable) generators of $\mathfrak{B},\{X, \mathfrak{B}, \mu\}$ is measure-isomorphic to $\left\{I^{R}, \tilde{\mathfrak{B}}, m\right\}$ and $\left\{T_{t}\right\}$ is mapped to the shift operator $\left\{S_{t}\right\}$. This $\left\{I^{\boldsymbol{R}}, \tilde{\mathfrak{B}}, m, S_{t}\right\}$ is called the symbolic repre- 
sentation of $\left\{X, \mathfrak{B}, \mu, T_{t}\right\}$. By this representation, the diversity of $X, \mathfrak{B}$ and $T_{t}$ is eliminated, and remains the arbitrariness of measure only. In other words, the diversity of flows is only the diversity of $\left\{m_{k_{1} k_{2} \cdots k_{n}}^{\left(t_{1} t_{2} \cdots t_{n}\right)}\right\}$ which satisfy (8.2).

Evidently, the similar (rather simpler) discussion holds for a discrete flow $\left\{T^{n}\right\}_{n=0, \pm 1, \pm 2, \cdots}$. This case (often assuming that $T$ is measurepreserving) has been studied in detail. Particularly, the studies on the existence of finite or countable generators are reviewed in [9].

In this paper, we generalized Kolmogorov's result to infinite measures, so that on an infinite measure space also we can consider the symbolic representation of flows.

Let $\left\{E_{k}\right\}_{1 \leqq k \leqq s}$ be a finite partition of $X$. Since $\mu$ is infinite, some $\mu\left(E_{k}\right)$ must be infinity. For simplicity, we assume $\mu\left(E_{1}\right)=\infty$ and $\mu\left(E_{k}\right)$ $<\infty$ if $k \neq 1$. Furthermore, we assume that transformation $T_{t}$ keeps the finiteness of measure, namely

$$
\mu(E)<\infty \Rightarrow \mu\left(T_{t} E\right)<\infty
$$

Then, (8.1) shows us that

$$
m_{k_{1} k_{2} \cdots k_{n}}^{\left(t_{1} t_{2} \cdots t_{n}\right)} \begin{cases}=\infty & \text { if } k_{1}=k_{2}=\ldots=k_{n}=1 \\ <\infty & \text { otherwise. }\end{cases}
$$

This is just the situation of $\S 7,2)$. So this $\left\{m_{k_{1} k_{2} \cdots k_{n}}^{\left(t_{1} t_{2} \cdots t_{n}\right)}\right\}$ can be extended to a $\sigma$-additive measure $m$ on $I^{\boldsymbol{R}}$, and we get the symbolic representation of $\left\{X, \mathfrak{B}, \mu, T_{t}\right\}$.

If $\left\{E_{k}\right\}_{1 \leqq k<\infty}$ is a countable partition of $X$, all $\mu\left(E_{k}\right)$ may be finite. Assuming that at most one of $E_{k}$ has infinite measure, we can reduce the problem to the discussion in $\S 7,3$ ).

Thus, on an infinite measure space also, the diversity of flows comes from only the diversity of $\left\{m_{k_{1} k_{2} \cdots k_{n}}^{\left(t_{1} t_{2} \cdots t_{n}\right)}\right\}$ which satisfy (8.2) and (8.6). (For countable case, the first line in (8.6) is not necessary).

As mentioned in $\S 7$, the family $\left\{m_{k_{1} k_{2} \cdots k_{n}}^{\left(t_{1} t_{2} \cdots t_{n}\right)}\right\}$ does not determine its extension on $\tilde{\mathfrak{B}}$ uniquely. If we assume that the flow is continuous (on the metric space defined by $\rho(E, F)=\mu(E \ominus F)$ ), the extension is determined uniquely if we determine the value 


$$
c=m\left(\bigcap_{r \in Q} p_{r}^{-1}(\{1\})\right)
$$

where $Q=$ the set of all rational numbers. If $\left\{m_{k_{1} k_{2} \cdots k_{n}}^{\left(t_{1} t_{2} \cdots t_{n}\right)}\right\}$ satisfies $(7.7)^{\prime}$ or $(7.8)^{\prime}, c$ may be any value, but otherwise $c$ must be infinity.

Assume that $\left\{T_{t}\right\}$ is ergodic. Namely if $E$ is $T_{t}$-invariant modulo null-set, $E$ or $X-E$ has zero measure. Under this assumption, $c$ must be zero, because $\bigcap_{r \in Q} T_{r} E_{1}$ is $T_{t}$-invariant modulo null-set and disjoint with $E_{k}(k \neq 1)$ which is naturally supposed not to be a null set. Thus, an ergodic flow corresponds to the smallest extension of $\left\{m_{k_{1} k_{2} \cdots k_{n}}^{\left(t_{1} t_{2} \cdots t_{n}\right)}\right\}$.

Even if $\left\{T_{t}\right\}$ is not ergodic, any ergodic part of it corresponds to the smallest extension. Similarly, even if $\mathfrak{B}$ has not finite (or countable) generators, the restriction of the flow $\left\{T_{t}\right\}$ on $\mathfrak{B}^{\prime}$ (=the smallest $\sigma$-ring generated by $\left\{T_{t} E_{k}\right\}$ ) allows the symbolic representation. These considerations may be useful for the decomposition of $\left\{T_{t}\right\}$ as the sum of symbolic representations.

Remark For an infinite measure space also, the existence of finite generators is assured by the Theorem 2.4 in [9].

\section{References}

[1] Kolmogorov, A. N., Grundbegriffe der Wahrscheinlichkeitsrechnung. Springer (1933).

[2] Bochner, S., Harmonic analysis and the theory of probability. Univ. of California (1955).

[3] Parthasarathy, K. R., Probability measures on metric spaces. Academic Press (1967).

[4] Okabe, Y., On Kolmogorov's extension theorem. Sügaku. 20 No. 3 (1968) 222-225 (in Japanese).

[5] Umemura, Y Measures on infinite dimensional vector spaces. Publ. RIMS, Kyoto Univ. 1 No. 1 (1965) 1-47.

[6] Umemura, Y. and Kôno, N., Infinite dimensional Laplacian and spherical harmonics. Publ. RIMS, Kyoto Univ. 1 No. 2 (1966) 163-186.

[7] Hida, T. and Nomoto, H., Gaussian measure on the projective limit space of spheres. Proc. Japan Acad. 40 No. 5 (1964) 301-304.

[8] Shale, D. and Stinespring, W. F., Integration over non-Euclidean geometries of infinite dimension. J. Math. Mech. 16. No. 2 (1966) 135-146.

[9] Krengel, U., Recent results on generators in ergodic theory, Transactions of Sixth Prague Conference on Information Theory, Statistical Decision Functions and Random Processes. (held 1971, published 1973) 465-482. 
